\title{
SUPERCONDUCTING SOLENOIDS FOR THE MUON COILIDER
}

\author{
M. A. Green \\ Lawrence Berkeley National Laboratory \\ Y. Eyssa, S. Kenny, J.R. Miller, S. Prestemon \\ National High Magnetic Field Laboratory \\ R. J. Weggel \\ Brookhaven National Laboratory
}

December 1999

Supported by U.S. Department of Energy under Contract Nos. DE-ACO3$76 S F 00098$ and $D E-A C O 2-98 C H 10886$. 


\title{
Superconducting Solenoids for the Muon Collider
}

\author{
M. A. Green(a) Y Eyssa(b), S. Kenny(b), J R. Miller(b), S. Prestemon(b), \\ and R. J Weggel(c) \\ (a) Lawrence Berkeley National Laboratory, Berkeley CA, USA, \\ (b) National High Magnetic Field Laboratory, Tallahassee FL, USA, \\ (c) Brookhaven National Laboratory, Upton NY, USA
}

\section{September 1999}

Submitted to the 16th International Conference on Magnet Technology Ponte Verde Beach, Florida, 27 September through 1 October 1999

* This work was performed ath the Lawrence Berkeley National Laboratory with the support of the Office of Science, United States Department of Energy under contract numbers DE-AC03-76SF00098. 


\title{
Superconducting Solenoids for the Muon Collider*
}

\author{
M. A. Green(1) Y. Eyssa( ${ }^{(2)}$, S. Kenny ${ }^{(2)}$, J. R. Miller ${ }^{(2)}$, S. Prestemon(2), and R. J. Weggel(3) \\ (1) Lawrence Berkeley National Laboratory, Berkeley CA, USA, \\ (2) National High Magnetic Field Laboratory, Tallahassee FL, USA, \\ (3) Brookhaven National Laboratory, Upton NY, USA
}

\begin{abstract}
The muon. collider is a new idea for lepton colliders. The ultimate energy of an electron ring is limited by synchrotron radiation. Muons, which have a rest mass that is 200 times that of an electron can be stored at much higher energies before synchrotron radiation limits ring performance. The problem with muons is their short life time $(2.1 \mu s$ at rest). In order to operate a muon storage ring large numbers of muon must be collected, cooled and accelerated before they decay to an electron and two neutrinos. As we see it now, high field superconducting solenoids are an integral part of a muon collider muon production and cooling systems. This report will describe the design parameters for superconducting and hybrid solenoids that are used for pion production and collection, RF phase rotations of the pions as they decay into muons and the muon cooling (reduction of the muon emittance) before acceleration.
\end{abstract}

\section{BACKGROUND}

A muon collider is one of several candidates for a next generation high energy physics machine. Muons are electron like particles that have a rest mass that is 200 times greater than that of the electron. Muons do not produce the synchrotron radiation that is produced by electrons when they are bent in a magnetic field. This means that muons can be stored in a circular ring at much higher energies than electrons. The muon collider can potentially extend the collision range for electron like particles without having linear accelerators that are very long (many kilometers). The problem is that muons are short lived particles that are only produced from pions produced by targeting protons. The acceleration and storage of the muons must occur before they decay into other particles.

The proposed muon collider consists of the following components[1,2]: 1) a proton driver that accelerates a large number of protons, 2) a hybrid solenoid used to capture pions produced by the protons striking the target, 3) a decay and phase rotation channel for the positive and negative pions produced at the target, 4) a system to cool the muons (reduce their emittance) prior to acceleration to high energies, 5) a series of accelerators to accelerate the muons before they decay, 6) a muon storage ring that stores muons at energies up to $2 \mathrm{TeV}$ long enough to produce a significant number of collisions for analysis, and 7) a detector that detects the particles produced by the collision of positively and negatively charged muons in the storage ring.

Manuscript was received 27 September 1998.

* The support for this work came from the Office of Science, United States Department of Energy under contracts DE-AC0376 SF00098.
Solenoidal magnetic fields will be used in the muon collider for the following tasks: 1) Pions produced from a tilted target hit by the proton beam will be captured in a $20 \mathrm{~T}$ hybrid solenoid. 2) Solenoids will be used to guide and focus the pions as they decay to muons in a channel that is about 40 meters long. While the pions decay to muons, the cloud of pions and muons must be phase rotated into a bunched beam, using RF cavities. 3) Muons will be cooled (their transverse and longitudinal emittance will be reduced) in a channel of alternating field solenoids. A schematic illustration of the pion capture system, the phase rotation system and the muon cooling system is shown in Figure 1.

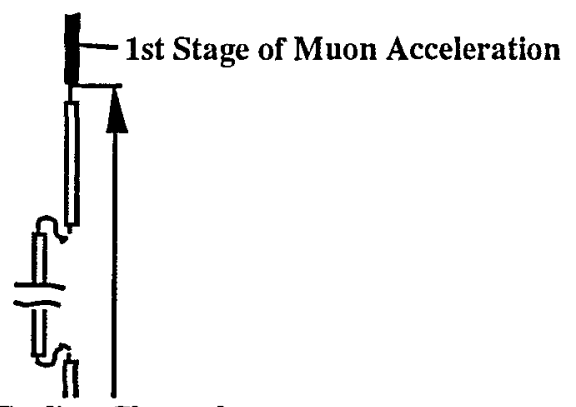

Muon Cooling Channel

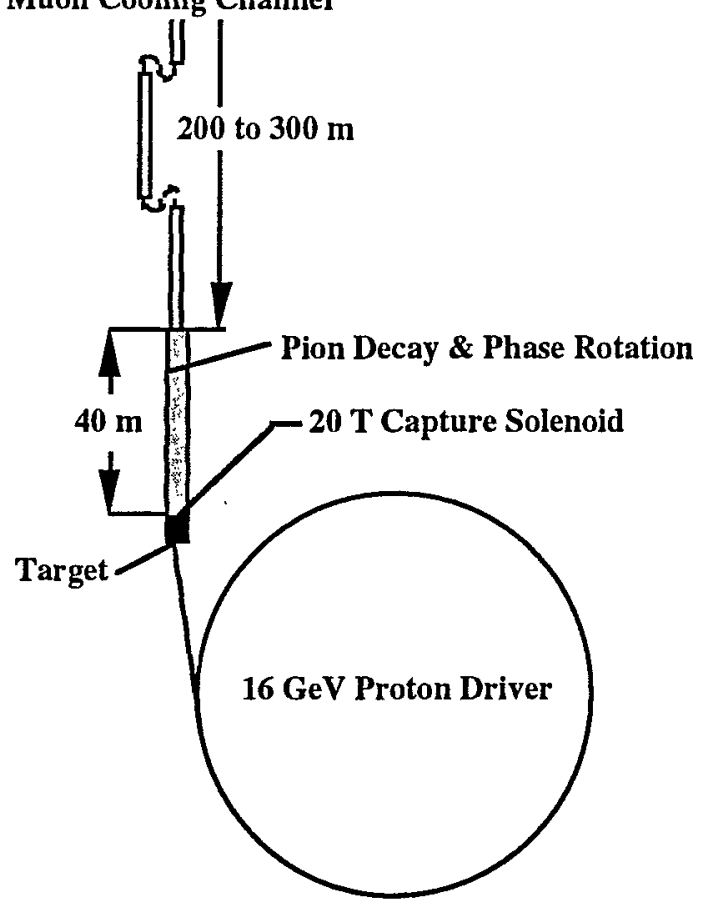

Fig. 1 A schematic Representation of the Muon Production and Cooling System for a Muon Collider 


\section{CAPTURE SOLENOID SYSTEM}

Capture of the pions from the target can occur in a solenoid of any induction provided the solenoid bore is large enough [3,4]. A capture induction of $20 \mathrm{~T}$ in a radius of 75 $\mathrm{mm}$ is needed to capture pions with a transverse momentum up to $225 \mathrm{MeV} / \mathrm{c}$. Once the pions have been captured in the solenoidal field, the channel solenoidal induction is reduced to about $1.25 \mathrm{~T}$. Pion decay and phase rotation will occur at this level of magnetic induction. The beam radius increases from $75 \mathrm{~mm}$ to $300 \mathrm{~mm}$ as the magnetic induction changes from $20 \mathrm{~T}$ to $1.25 \mathrm{~T}$

The hybrid magnet option was selected for the following reasons: 1) The operating power for the water cooled solenoid is substantially lower if a superconducting outsert is used. About $14 \mathrm{~T}$ of the $20 \mathrm{~T}$ capture solenoid induction is produced by the superconducting solenoids. 2) The current density in the water cooled solenoid must be low enough to insure that its life time will be long. (The life time goal is over 25000 hours.) 3) The water cooled solenoid and a water cooled liner in the hybrid magnet absorbs the energy from particles not captured by the capture solenoid system (most of the $4 \mathrm{MW}$ of beam power deposited on the target). The water cooled insert solenoid and water cooled absorber must limit the heat flux into the superconducting magnets to $100 \mathrm{~W}$ or less. Figure 2 shows a schematic representation of a capture solenoid system around a tilted target.

The superconducting outsert solenoid system could consist of three coils. The outermost coil would be made from niobium titanium while the system of inner coils would be made from niobium tin. If $1.8 \mathrm{~K}$ refrigeration is used the niobium titanium solenoid could generate $8.5 \mathrm{~T}$ while the two inner solenoids would generate up to 6.5 T. Cable in conduit technology may be particularly attractive for the hybrid capture solenoid magnet $[5,6]$

The conventional insert solenoid would be made using copper with a ceramic insulation system. The high level of radiation damage in the inner solenoid is particularly troublesome. The use of Bitter type solenoid as an insert was considered but rejected because the demonstrated life time of this type of magnet is too short.

The niobium titanium transfer solenoid system guides the magnetic field so that the magnetic induction decreases in a smooth fashion from $20 \mathrm{~T}$ to $1.25 \mathrm{~T}$ at the start of the phase rotation channel. A smooth transition of the magnetic induction can be achieved if the magnetic induction $\mathrm{B}(\mathrm{x})$ as a function of the distance from the end of the target $x$ has the following relationship;

$$
B(x)=\frac{B o}{x+A}
$$

where $B_{0}$ and $A$ are fitting parameters. If one reduces the magnetic field from $20 \mathrm{~T}$ to $1.25 \mathrm{~T}$ in $2.5 \mathrm{~m}, \mathrm{~B}_{\mathrm{O}}=3.333 \mathrm{Tm}$ and $A=0.1667 \mathrm{~m}$. Once the magnetic induction has reached the design value for phase rotation or pion beam transport, it is desirable for the induction to remain at a constant value. The diameter of the beam region increases from $150 \mathrm{~mm}$ to $600 \mathrm{~mm}$ as the solenoid induction drops to $1.25 \mathrm{~T}$.

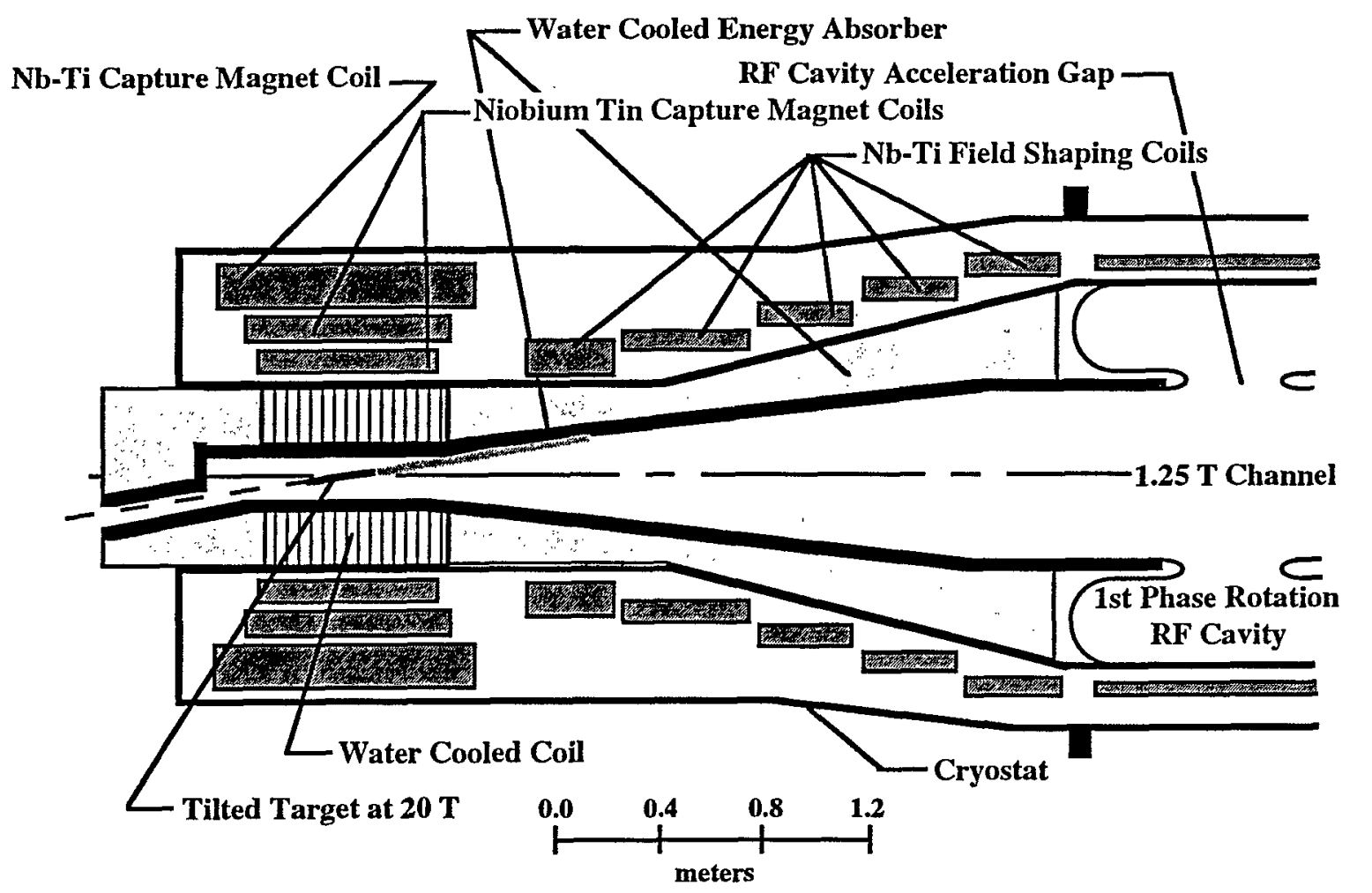

Fig. 2. A Cross-section of a 20 T Hybrid Capture Solenoid for Pions Produced in a Tilted Solid Target. 


\section{PHASE ROTATION AND DECAY SOLENOIDS}

Once the pions have been captured in a solenoid field they must be transferred to a channel where they decay into muons. RF cavities are required to keep the pions bunched as they decay to muons. The momentum spread of the captured pions entering the channel is from $100 \mathrm{MeV} / \mathrm{c}$ to $800 \mathrm{MeV} / \mathrm{c}$. The lower momentum muons will lag behind the high momentum muons as they travel down the channel. The role of the RF cavities is to bunch the muons in both physical and phase space by accelerating the slow muons and decelerating the fast muons. The muons should be kept in well defined bunches that travel at the same velocity down the channel.

A $200 \mathrm{MeV}$ pion decays to a muon in about $4.2 \times 10^{-8} \mathrm{~s}$. Virtually all of the pions decay to muons and low energy neutrinos. Ninety-seven percent of the pions are converted to muons within 40 meters of the production target. Ideally muon cooling should start at or near this point, where the muon flux reaches its maximum.

Phase rotation is done in a series of RF cavities that have a frequency of about $90 \mathrm{MHz}$ at the start of the channel. At the end of the channel, the frequency drops to about $60 \mathrm{MHz}$. In order to prevent beam loss in the phase rotation channel, the solenoidal field within the RF cavities must be uniform to one or two percent. As a result, the phase rotation solenoids can't be inside of the RF cavity. A large gap between solenoids produces a non-uniform solenoidal field.

A $90 \mathrm{MHz}$ RF cavity will fit into a solenoid that has a warm bore diameter of $1.6 \mathrm{~m}$. As the $\mathrm{RF}$ frequency goes down along the phase rotation channel, the magnet warm bore size must be increased to about $2.4 \mathrm{~m}$. Gaps in the solenoids for RF cavity services will not cause field non-uniformity, if there is extra conductor around the gap. Figure 3 shows a schematic representation of a phase rotation solenoid at its largest diameter at the end of the channel.
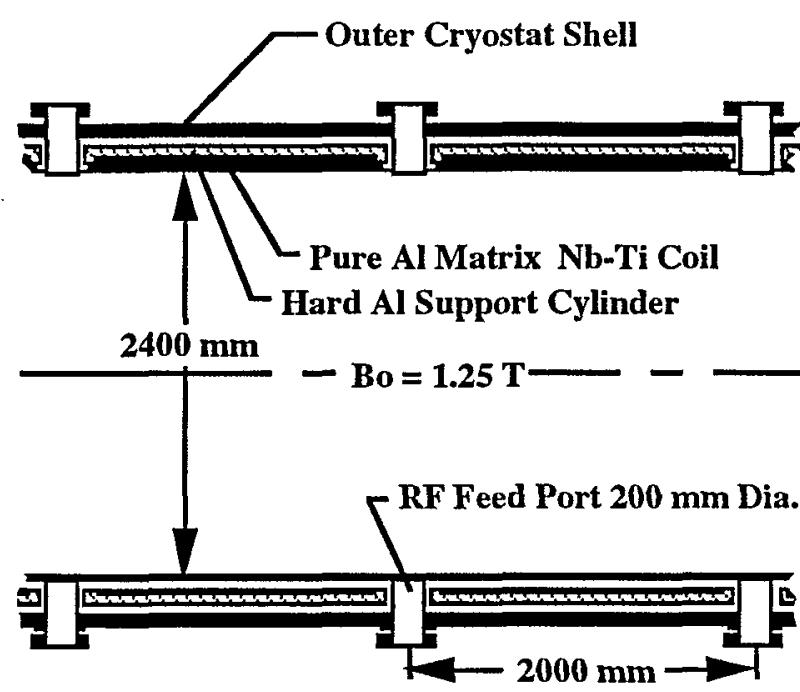

Fig. 3 A Schematic Representation of a Phase Rotation Solenoid for the Low-Frequency End of the Phase Rotation System
There is a trade-off between the cost of the magnets, the cost of the cavities and the RF power system. Larger diameter RF cavities will require less power per unit acceleration gradient than cavities that are smaller. The cost of the RF driver is proportional to the RF power needed to drive the RF cavities. The cost of the superconducting solenoid around the RF cavities can be estimated using the following relationship[7]:

$$
\text { Cost }=0.126 \mathrm{D}^{1.29} \mathrm{~B}^{1.4}
$$

where the cost is given in M\$ per meter of solenoid length; $\mathrm{D}$, the solenoid warm bore diameter, is given in meters; and $B$, the solenoid central induction, is given in tesla.

For the magnet systems that have been studied, the stored energy per unit length is about $1.25 \mathrm{MJ} / \mathrm{m}$ at the start of the channel, and it goes up to about $2.81 \mathrm{MJ} / \mathrm{m}$ at the end of the channel. For a phase rotation channel that is $40 \mathrm{~m}$ long, the stored energy of the superconducting guide field solenoids would be about $80 \mathrm{MJ}$. The phase rotation channel solenoid could be built in 10 meter long sections that increase in diameter to fit around the RF cavities.

\section{HIGH FIELD MUON COOLING SOLENOIDS}

The muon cooling system will have a series of solenoids that produce increasing induction as one proceeds down the cooling channel. The central induction at the start of the channel will be about $1.25 \mathrm{~T}$ in a region that is $600 \mathrm{~mm}$ in diameter. Cooling occurs when the muon beam is focused within a container of liquid hydrogen that is about $400 \mathrm{~mm}$ long. A length of $400 \mathrm{~mm}$ of liquid hydrogen reduces the energy of a $160 \mathrm{MeV}$ muon by about $25 \mathrm{MeV}$. The muon energy is reduced in both the transverse and the longitudinal directions. Between the cooling sections are RF cavities that accelerate the beam in the longitudinal direction by $25 \mathrm{MeV}$ without adding transverse momentum.

The frequency of the RF cavities starts at the RF frequency of the phase rotation system. As one goes down the cooling channel, the RF frequency can be increased, bunching the muon beam as it is cooled. The acceleration gradient in the RF cavity is proportional to the square root of the RF frequency. In the cases studied, the RF frequency can start at $60 \mathrm{MHz}$ and go up to $805 \mathrm{MHz}$ in the region of the cooling channel, where the induction in the cooling section reaches $10 \mathrm{~T}$. The RF cavity induction starts at $1.25 \mathrm{~T}$, and it goes up to about $6 \mathrm{~T}$. At $805 \mathrm{MHz}$, the RF solenoids have a warm bore of $440 \mathrm{~mm}$.

In order to phase match the muon beam (so that no muons are lost) between one cooling section and the next, the magnetic flux must be reversed. Two cooling lattices have been studied. The first has flux reversal at low fields in the cavity. The second has flux reversal in the hydrogen absorber. The advantage of reversing the flux in the absorber is that the highest fields in the channel will be lower than for the case where flux reversal occurs in the cavities (16 T versus about $30 \mathrm{~T}$ ). Figures 4 and 5 and Table 1 shows the parameters of the two types of cooling schemes that have been studied to date. 


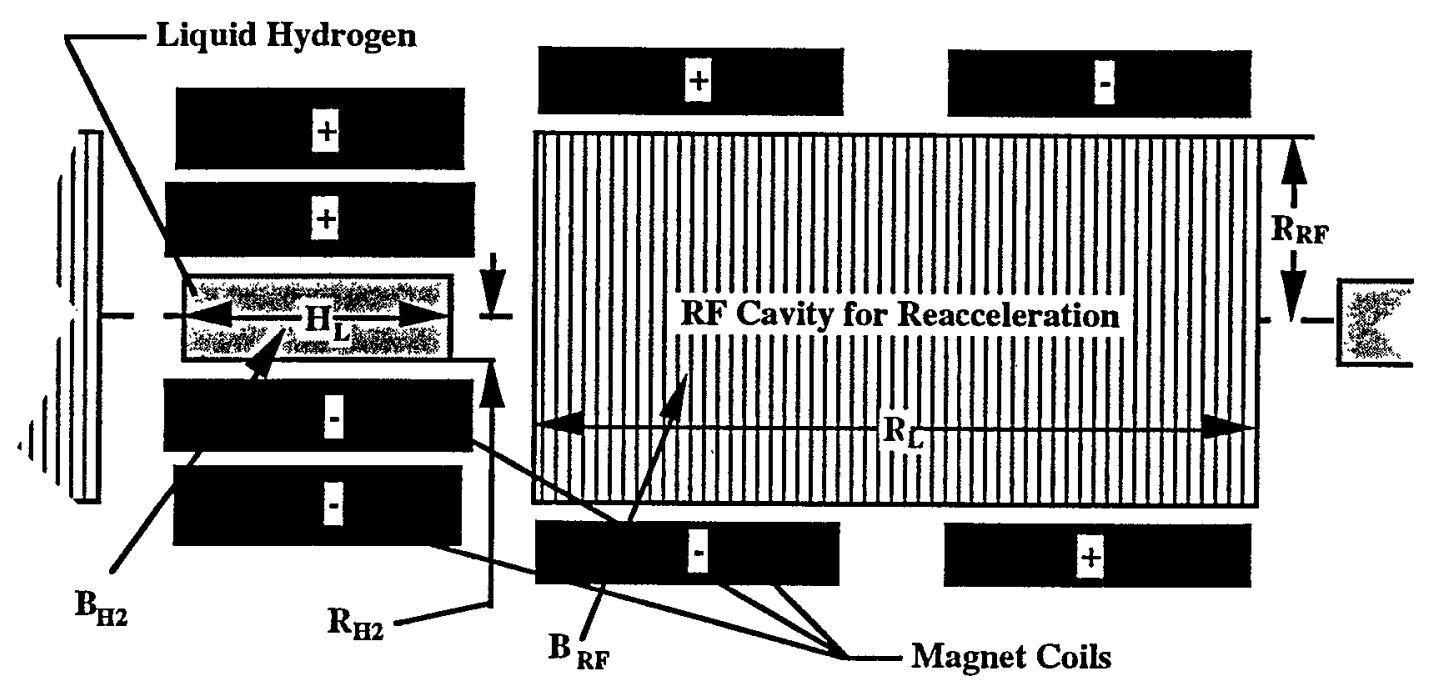

Figure 4 A Typical Standard Muon Cooling Cell with Flux Reversal in the RF Cavity (See Table 1)

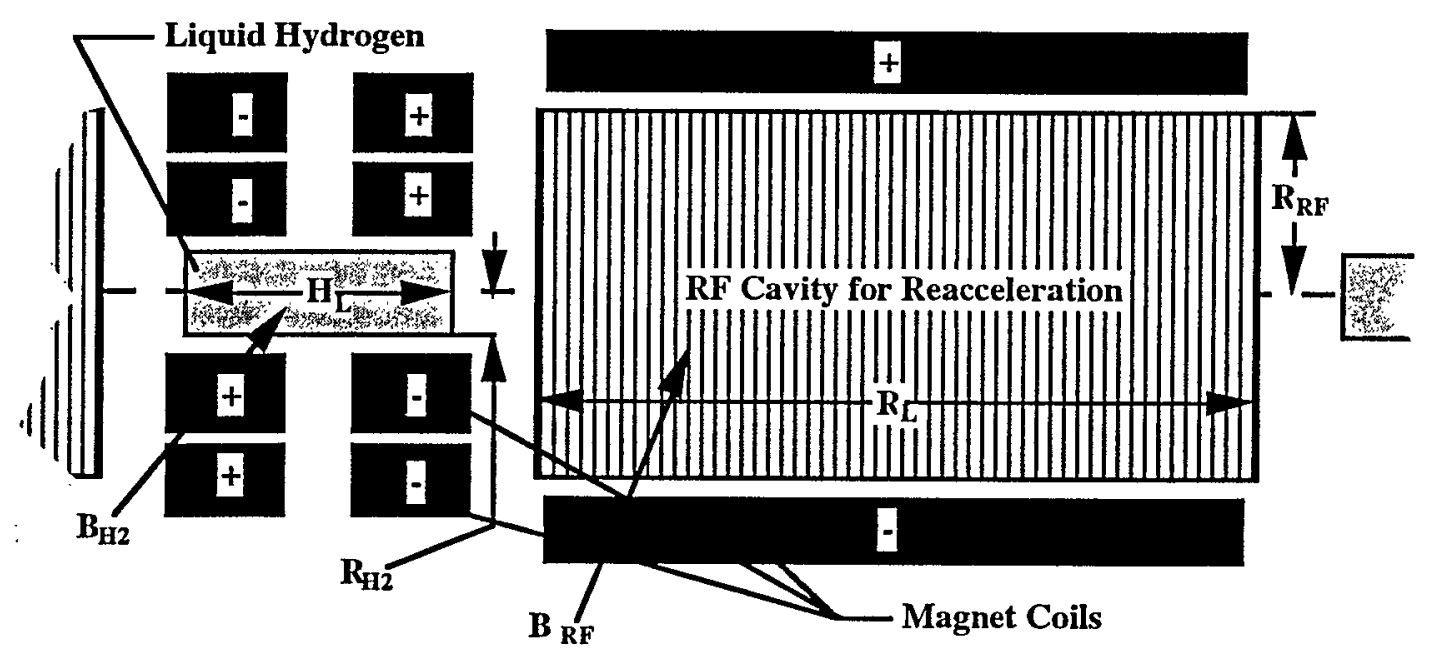

Figure 5 A Typical Alternative Muon Cooling Cell with Flux Reversal in the Hydrogen Absorber (See Table 1)

Table 1 Parameters for Muon Cooling Cells for the Standard and Alternative Cooling Lattices as a Function of the Induction in the Hydrogen Muon Energy Absorber

$\begin{array}{cccccccc}\mathrm{B}_{\mathrm{H} 2} & \text { Beam Radius } & \mathrm{H}_{2} \text { Radius } & \mathrm{H}_{2} \text { Length } & \mathrm{B}_{\mathrm{RF}} & \text { RF Frequency } & \text { RF Radius } & \text { RF Length } \\ (\mathrm{T}) & \mathrm{R}_{\mathrm{b}}(\mathrm{mm}) & \mathrm{R}_{\mathrm{H} 2}(\mathrm{~mm}) & \mathrm{H}_{\mathrm{L}}(\mathrm{mm}) & (\mathrm{T}) & (\mathrm{MHz}) & \mathbf{R}_{\mathrm{RF}}(\mathrm{mm}) & \mathrm{R}_{\mathrm{L}}(\mathrm{mm})\end{array}$

\section{Standard Muon Cooling Mode}

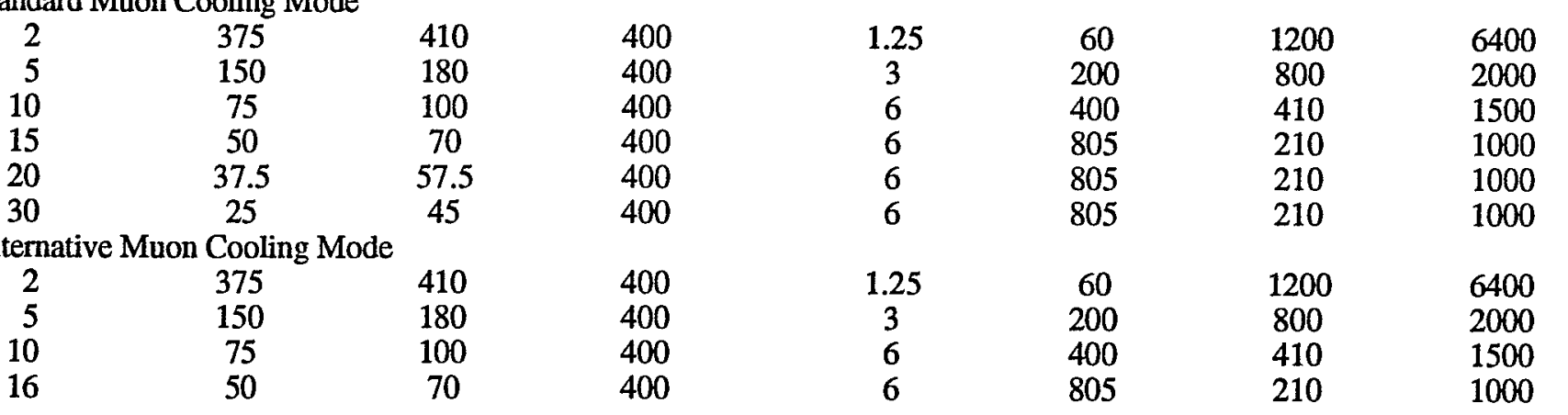




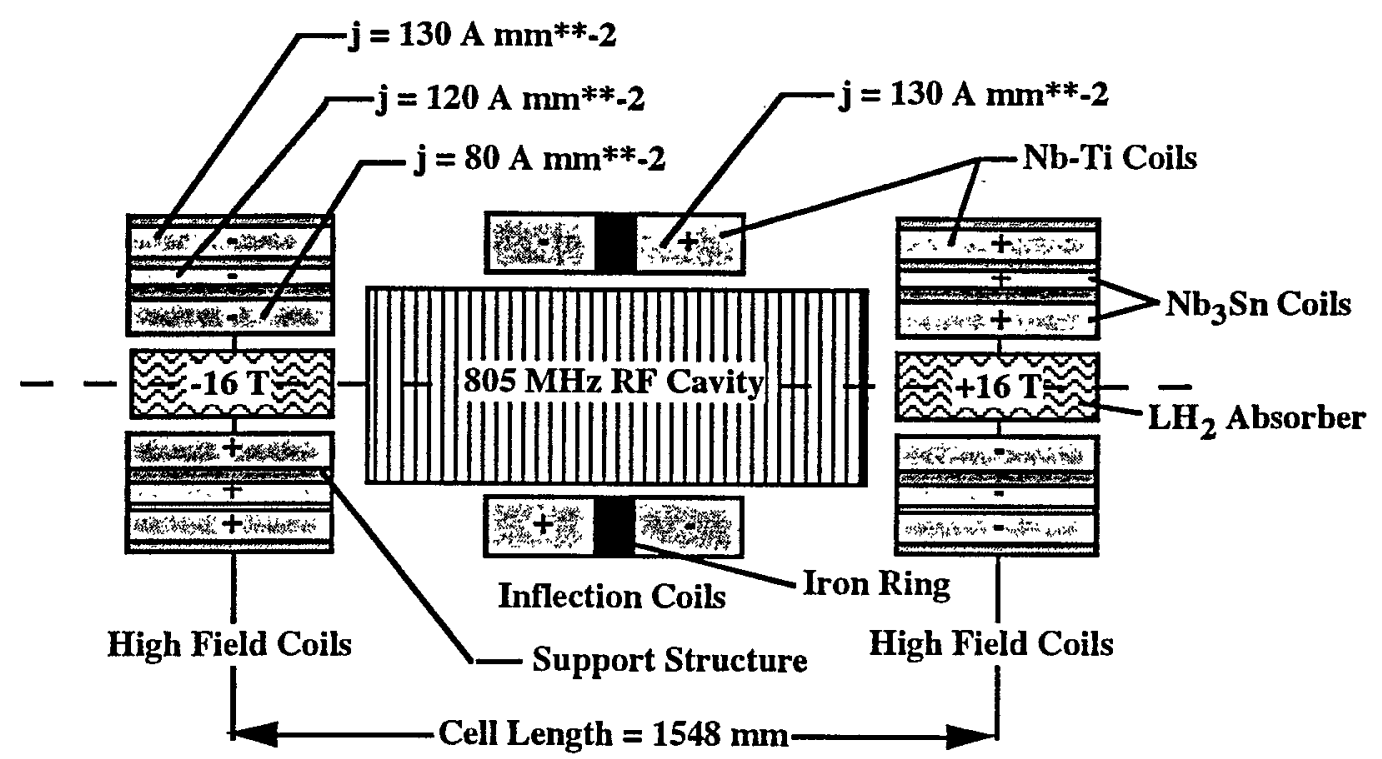

Figure 6 A Standard Muon Cooling Cell with $16 \mathrm{~T}$ at the Center of the Hydrogen Absorber (See Figure 7)

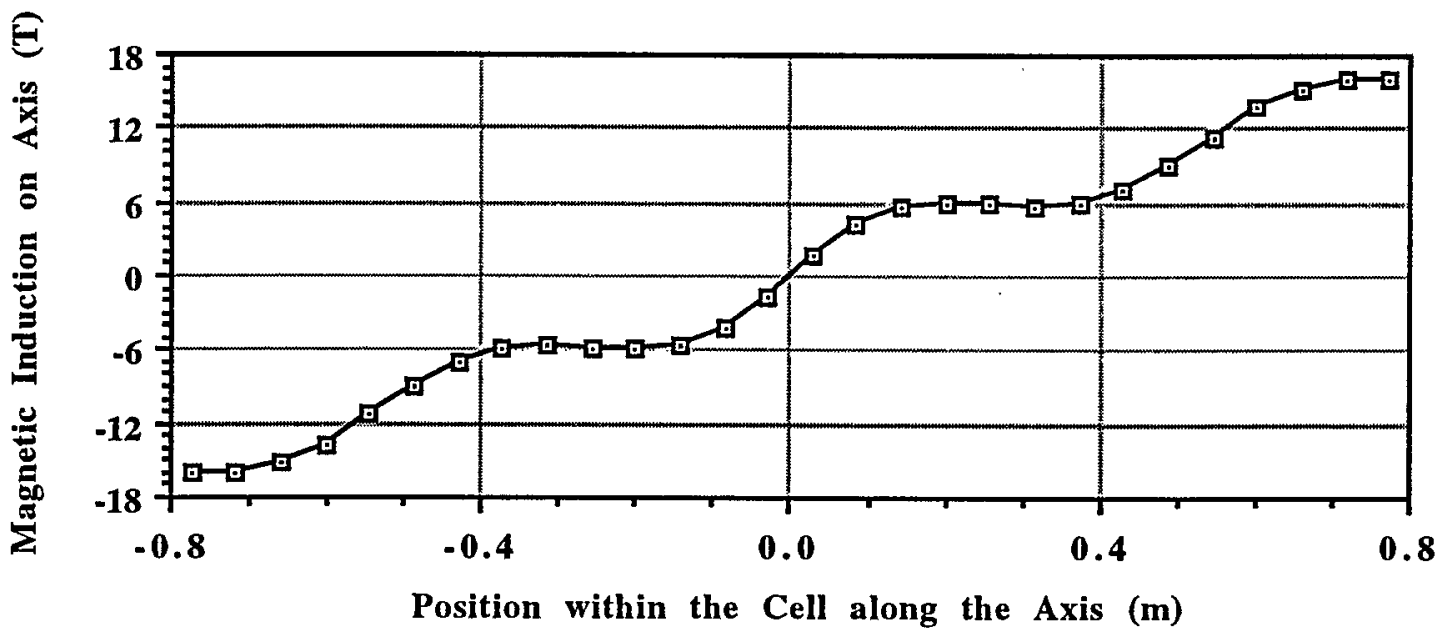

Figure 7 The Field Profile Created by the 16 T Standard Cooling Cell Shown in Figure 6

The standard cell[8] shown in Figure 4 has the field reverse in the RF cavity. Field gradients in the RF cavity may affect the acceleration gradient in the RF cavity. During the year 2000 an $805 \mathrm{MHz}$ high gradient RF cavity will be tested in a gradient field to determine the extent of the problem. The test solenoid magnet that will be used for the RF cavity experiment is described in Ref. [9]. As a comparison, the RF cavity will be operated in a constant magnetic field and with no magnetic field.

The exact field profile in the muon cooling cell is important if the muons are to remain captured as they move from cooling cell to cooling cell. The field profile generated by the magnet configuration shown in Figure 6 is shown in Figure 7. The dip in the magnitude of the magnetic induction between the high field coils and the gradient coils is necessary, if the muon beam is to remain captured in the cooling channel over a range of muon energies from 135 to $175 \mathrm{MeV}$.
The alternative cooling lattice shown in Figure 5 will cool the muons further without going to magnetic inductions as high as $30 \mathrm{~T}$ in the absorber. The same level of muon cooling can be achieved with an induction of 14 to $16 \mathrm{~T}$ in the hydrogen absorbers. The RF cavities are in a relatively uniform magnetic field in this scenario. The problems with the alternative cooling lattice are: 1) The flux reversal occurs in the high field magnet. Even though the diameter of the high field solenoid is relatively small, the forces pushing the high field coils apart are large. 2) High current densities are required in all of the magnet coils. Stress strain and training are real problems in the alternative lattice. The high field coils must be subdivided, and force carrying members must be provided when the lattice is at field above about $10 \mathrm{~T}$. 3) The alternative cooling channel is longer than the standard channel, so more muons will be lost during the cooling process. The alternative cooling channel may be more useful for a neutrino factory. 


\section{BENT SOLENOIDS FOR MOMENTUM MATCHING AND LONGITUDINAL MUON COOLING}

Every 20 meters the cooled muon beam emittance must be exchanged in momentum space. In order to get a spread of muons of various momenta, one has to bend the muon beam in a curved solenoid with an impressed dipole field. After the muon beam has been bent by the solenoid, muons of differing momenta are sorted in physical space across the diameter of the solenoid. Lithium wedges can be put into the bore of the solenoid so that high momentum muons will lose more energy than low momentum muons. A schematic of the bent solenoid momentum compaction system is shown in Fig. 8

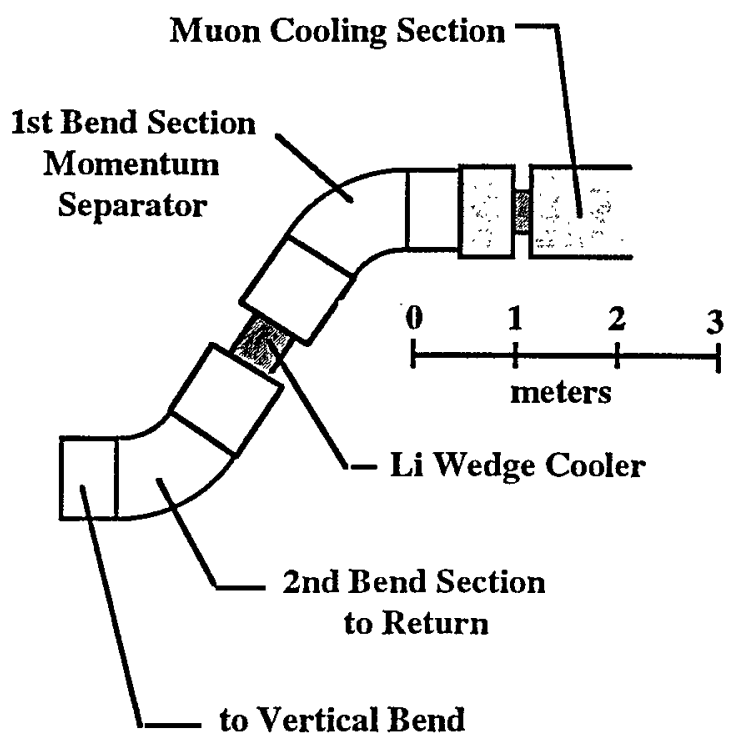

Figure 8 A Bent Solenoid for Momentum Compaction (Wedge) Cooling in the Horizontal Plane

The bent solenoid system shown in Fig. 8 can have a coil diameter up to $480 \mathrm{~mm}$. The larger coil diameter matches the RF cavities needed to accelerate the muon beam after the muons have lost momentum. The radius of the bend shown in Fig. 8 has a gradual change to a minimum bend radius of $1000 \mathrm{~mm}$. The variable radius of curvature insures that muon will stay within the cooling channel.

The design induction is set at $3.0 \mathrm{~T}$ at the axis of the bent solenoid. A dipole field is generated by tilting the coils used to make the bent solenoid. The tilt angle varies with the local radius of curvature of the bent solenoid[10,11]. The dipole induction varies across the bent solenoid in the same way the solenoidal induction varies (as 1 over $R$ ) across the solenoid. This dipole brings the muons separated by momentum to a plane at the center of the solenoid where the wedges are located. The dipole field at the center of the bent solenoid should be about $0.55 \mathrm{~T}$.

\section{CONCLUDING COMMENTS}

The muon collider has superconducting solenoids as an integral part of the first 250 to 350 meters of the machine. The solenoids serve both as a guide field and focusing elements during the time when the pions and muons have a very high emittance. Capture of the pions with a transverse momentum as high as $225 \mathrm{MeV} / \mathrm{c}$ is done at $20 \mathrm{~T}$. The field in the solenoid is decreased to $1.25 \mathrm{~T}$ as the diameter of the channel carrying the pions is increased by a factor of four. Phase rotation and pion decay to muons is done within a string of RF cavities at a solenoidal induction of $1.25 \mathrm{~T}$.

Muon cooling (reducing the six-dimensional muon emittance) is done at relatively low fields (about $2 \mathrm{~T}$ ) at the start of the cooling channel. As the muons are cooled and become more tightly bunched, the peak magnetic induction in the cooling channel must go up. At the high field end of the cooling channel, the induction can be in the range from $14 \mathrm{~T}$ to $30 \mathrm{~T}$ depending on the type of muon cooling channel.

\section{ACKNOWLEDGMENTS}

This work was performed with the support of the Office of Science, United States Department of Energy under contract numbers DE-AC03-76SF00098.

\section{REFERENCES}

[1] The $\mu^{+} \mu^{-}$Collider Collaboration, $\mu^{+} \mu^{-}$Collider, A Feasibility Study," BNL-52503, July 1996

[2] R. B. Palmer for the Muon Collider Collaboration "Muon Collider: Introduction and Status," BNL-65241, Jan.1998

[3] N. V. Mokhov, R. J. Noble and A. Van Ginneken, Targetry and Collection Optimization for Muon Colliders, Proceedings of the 9th Advanced ICFA Beam Dynamics Workshop, Montauk, NY, 15-20 Oct. 1995

[4] M. A. Green and R. J. Weggel, "A 20 T Hybrid Solenoid for the Collection of Pions for the Muon Collider," IEEE Trans. Appl. Supercon .7, No. 2, p 642, (1997)

[5] J. R. Miller, M. D. Bird, S. Bole, et al, "An Overview of the 45T Hybrid Magnet System for the National High Field Magnet Laboratory," IEEE Trans. Mag 30, No. 4, p 1563, (1994)

[6] Y. Iwasa, M. J. Leupold, R. J. Weggel, and J. E. C. Williams "Hybrid III: The System, Test Results, the Next Step," IEEE Trans. Appl. Supercon. 3, No. 1, p 58, (1993)

[7] M. A. Green and S. J. St Lorant, "Estimating the Cost of Large Superconducting Thin Solenoid Magnets," Advances in Cryogenic Engineering 39, p 271, Plenum Publishing, New York, 1993)

[8] M. A. Green et al, "High Field Superconducting Solenoids for Muon Cooling," submitted to EUCAS-99 Proceedings, Sitges, Spain, 14 to 17 September 1999

[9] M. A. Green, J. Y. Chen and S. T. Wang, "The Design, and Construction of a Gradient Solenoid for the High Power RF Cavity Experiment for the Muon Collider," submitted to EUCAS-99 Proceedings, Sitges, Spain, 14 to 17 September 1999

[10] M. A. Green et al, "Bent Superconducting Solenoids for the Muon Cooling Experiment" Particle Accelerator Conference Proceedings, p 3227, IEEE Publications, Piscataway NJ (1999)

[11] M. A. Green et al, "Bent Solenoid Simulation for the Muon Cooling Experiment to be published in Advances in Cryogenic Engineering 45, Plenum Press (1999) 